\title{
ROUGH AND STRONGLY ROUGH NORMS ON BANACH SPACES
}

\author{
G. GODINI
}

\begin{abstract}
We give equivalent conditions for a norm on a Banach space $X$ to be rough using the sets $A(x)$ defined for each $x \in X$ by $A(x)=\left\{f \in X^{*} \mid f(x)=\|x\|\right.$, $\|f\|=1\}$. This enables us to obtain unitary characterizations for rough and strongly rough norms.
\end{abstract}

Let $X$ be a real Banach space, and for $x, y \in X$, let $\tau(x, y)$ denote the one sided Gateaux differential of $\|\cdot\|$ at $x$ in the direction $y$, i.e.,

$$
\tau(x, y)=\lim _{t \rightarrow 0^{+}} t^{-1}(\|x+t y\|-\|x\|) .
$$

For $x_{0} \in X$ and $r>0$ we denote $B_{X}\left(x_{0}, r\right)=\left\{x \in X \mid\left\|x-x_{0}\right\| \leqslant r\right\}, B_{X}=$ $B_{X}(0,1)$ and $S_{X}=\{x \in X \mid\|x\|=1\}$. For each $x \in X$ let us denote $A(x)=A_{X}(x)$ $=\left\{f \in S_{X^{*}} \mid f(x)=\|x\|\right\}$, where $X^{*}$ is the dual space of $X$. For $f \in S_{X^{*}}$, let $A^{-1}(f)=\left\{x \in S_{X} \mid f(x)=1\right\}$ and denote by $\operatorname{Dom}\left(A^{-1}\right)$ the set $\left\{f \in S_{X^{*}} \mid A^{-1}(f) \neq\right.$ $\varnothing\}$.

By the Ascoli-Mazur Theorem [4, p. 447], for $x, y \in X$ we have

$$
\tau(x, y)=\max \{f(y) \mid f \in A(x)\} \text {. }
$$

DEFINITION $1[8,9$, p. 120]. A norm of a Banach space $X$ is said to be rough if there is an $\varepsilon>0$ such that for every $x \in X$ and $\delta>0$, there exist $x_{1}, x_{2}, u \in X$, $\left\|x_{i}-x\right\|<\delta, i=1,2, u \in S_{X}$, with $\tau\left(x_{2}, u\right)-\tau\left(x_{1}, u\right) \geqslant \varepsilon$.

There are several known characterizations of a Banach space with a rough norm (see e.g., $[1,7,9,11,13])$. We add to these characterizations some new ones. In what follows we shall use the following results of $[7$, Proposition 1, (i) $\Leftrightarrow$ (iv), Proposition $1^{\prime}$, (ii) $\Leftrightarrow$ (iv)], some of them being implicitly contained in [9, p. 122].

Proposition 1 [7, p. 336]. The following properties of a given norm of $X$ are equivalent:

(i) The norm of $X$ (the dual norm of $X^{*}$ ) is not rough.

(ii) For every $\varepsilon>0$ there is an $x \in S_{X}\left(f \in S_{X^{*}}\right)$ such that whenever $f_{n}, g_{n} \in S_{X}$, $\lim f_{n}(x)=\lim g_{n}(x)=1\left(x_{n}, y_{n} \in S_{X}, \lim f\left(x_{n}\right)=\lim f\left(y_{n}\right)=1\right)$, then

$$
\lim \sup \left\|f_{n}-g_{n}\right\| \leqslant \varepsilon \quad\left(\lim \sup \left\|x_{n}-y_{n}\right\| \leqslant \varepsilon\right) .
$$

For a bounded set $A \subset X$, we denote the diameter of $A$ by $\operatorname{diam} A=\operatorname{diam}_{X} A=$ $\sup \left\{\left\|a_{1}-a_{2}\right\| \mid a_{1}, a_{2} \in A\right\}$.

Received by the editors October 19, 1981 and, in revised form, April 23, 1982.

1980 Mathematics Subject Classification. Primary 46B99. 
THEOREM 1. The following properties of a given norm of $X$ are equivalent:

(i) $\|\cdot\|$ is rough.

(ii) There is an $\varepsilon>0$ such that for every $x \in S_{X}$ and $\delta>0$, there exists $y \in S_{X}$, $\|y-x\|<\delta$ with $\operatorname{diam}(A(x) \cup A(y)) \geqslant \varepsilon$.

Proof. (i) $\Rightarrow$ (ii). Since $\|\cdot\|$ is rough, choose $\varepsilon>0$ given by Definition 1 , and let $\varepsilon^{\prime}=\varepsilon / 2, x \in S_{X}$ and $0<\delta<2$. By (i) for this $x \in S_{X}$ and $\delta / 2$, there exist $x_{1}, x_{2}, u \in X,\left\|x_{i}-x\right\|<\delta / 2, i=1,2, u \in S_{X}$, with $\tau\left(x_{2}, u\right)-\tau\left(x_{1}, u\right) \geqslant \varepsilon$. By (1) there exist $f_{i} \in A\left(x_{i}\right), i=1,2$, such that $\tau\left(x_{i}, u\right)=f_{i}(u), i=1,2$. Then

$$
\varepsilon \leqslant \tau\left(x_{2}, u\right)-\tau\left(x_{1}, u\right)=f_{2}(u)-f_{1}(u) \leqslant\left\|f_{1}-f_{2}\right\| .
$$

Since $x_{i} \neq 0$, let $y_{i}=x_{i} /\left\|x_{i}\right\|, i=1,2$. Let $f \in A(x)$. Then $2 \varepsilon^{\prime} \leqslant\left\|f_{1}-f_{2}\right\| \leqslant \| f_{1}-$ $f\|+\| f-f_{2} \|$ and so we can suppose (interchanging the indices if necessary) that $\varepsilon^{\prime} \leqslant\left\|f_{1}-f\right\| \leqslant \operatorname{diam}\left(A(x) \cup A\left(y_{1}\right)\right)$, i.e., we have (ii).

(ii) $\Rightarrow$ (i). Let $\varepsilon>0$ be given by (ii) and let $\varepsilon^{\prime}=\varepsilon / 2$ and $x \in S_{X}$. By (ii), for each $n$ there exists $y_{n} \in S_{X},\left\|y_{n}-x\right\|<1 / n$ with $\operatorname{diam}\left(A(x) \cup A\left(y_{n}\right)\right) \geqslant \varepsilon$. Let $f_{n}, g_{n} \in$ $A(x) \cup A\left(y_{n}\right)$ such that $\left\|f_{n}-g_{n}\right\| \geqslant 3 \varepsilon / 4$ for each $n$. Clearly, $\lim f_{n}(x)=\lim g_{n}(x)$ $=1$ and $\lim \sup \left\|f_{n}-g_{n}\right\|>\varepsilon^{\prime}$, whence by Proposition 1 , the norm is rough.

REMARK 1. Using Theorem 1, it is easy to show that the norm of $X$ is rough if and only if there is an $\varepsilon>0$ such that for every $x \in S_{X}, f \in A(x)$ and $\delta>0$, there exists $y \in S_{X},\|y-x\|<\delta$ with $\sup _{g \in A(y)}\|f-g\| \geqslant \varepsilon$.

COROLlARY 1. The following properties of a given norm of $X$ are equivalent:

(i) $\|\cdot\|$ is rough.

(ii) There is an $\varepsilon>0$ such that for every $x \in S_{X}$ and $\delta>0$, there exist $x_{1}, x_{2}, u \in S_{X}$, $\left\|x_{i}-x\right\|<\delta, i=1,2$, such that $\tau\left(x_{2}, u\right)+\tau\left(x_{1},-u\right) \geqslant \varepsilon$.

(iii) The same as (ii) but with $x_{1}=x_{2}$ or one of $x_{1}, x_{2}$ equals $x$.

Proof. (i) $\Rightarrow$ (ii). This follows by Definition 1, using the well-known fact [4] that $-\tau\left(x_{1}, u\right) \leqslant \tau\left(x_{1},-u\right)$.

(ii) $\Rightarrow$ (i). Using formula (1) we obtain that (ii) implies condition (ii) of Theorem 1, whence the norm is rough.

(i) $\Rightarrow$ (iii). By Theorem 1(i) $\Rightarrow$ (ii), there is an $\varepsilon^{\prime}>0$ such that for every $x \in S_{X}$ and $\delta>0$, there exists $y \in S_{X},\|y-x\|<\delta$ with $\operatorname{diam}(A(x) \cup A(y)) \geqslant \varepsilon^{\prime}$. Let $f, g \in$ $A(x) \cup A(y)$ such that $\|f-g\|>\varepsilon^{\prime} / 2$. Then there is an element $u \in S_{X}$ with $(f-g)(u) \geqslant \varepsilon^{\prime} / 2$. If $f \in A(x)$ and $g \in A(y)$ (similarly if $g \in A(x)$ and $f \in A(y)$ ), then by (1) we obtain $\varepsilon^{\prime} / 2 \leqslant f(u)+g(-u) \leqslant \tau(x, u)+\tau(y,-u)$. In a similar way, if $f, g \in A(x)$ or $f, g \in A(y)$ we obtain respectively $\tau(x, u)+\tau(x,-u) \geqslant \varepsilon^{\prime} / 2$ or $\tau(y, u)+\tau(y,-u) \geqslant \varepsilon^{\prime} / 2$. Therefore we have (iii) e.g., for $\varepsilon=\varepsilon^{\prime} / 2$.

Since (iii) $\Rightarrow$ (ii) is obvious, this completes the proof of the corollary.

Using a slight modification of [6, Lemma 2] we have

Lemma 1. Let $x, y, u \in S_{X}$. If $\tau(x, u)+\tau(y,-u)=\varepsilon>0$, then there is $a v \in S_{X}$ such that $\tau(x, v) \geqslant \varepsilon / 4$ and $\tau(y,-v) \geqslant(\varepsilon / 4)-\|x-y\| / 2$. 
Proof. By (1) there are $f \in A(x), g \in A(y)$ such that $(f-g)(u)=\varepsilon$. Let $w=u-(f+g)(u) x / 2$. Then $0<\|w\| \leqslant 2$. Let $v=w /\|w\|$. We have

$$
\tau(x, v) \geqslant f(v)=\frac{1}{\|w\|}\left(\frac{(f-g)(u)}{2}\right)=\frac{\varepsilon}{2\|w\|} \geqslant \frac{\varepsilon}{4} .
$$

Since $g \in A(y)$, we have $g(x) \geqslant 1-\|x-y\|$ and so

$$
g(-u)+\frac{(f+g)(u)}{2} g(x) \geqslant \frac{(f-g)(u)}{2}-\|x-y\| .
$$

Hence

$$
\begin{aligned}
\tau(y,-v) & \geqslant g(-v)=\frac{1}{\|w\|}\left(g(-u)+\frac{(f+g)(u)}{2} g(x)\right) \\
& \geqslant \frac{1}{\|w\|}\left(\frac{\varepsilon}{2}-\|x-y\|\right) \geqslant \frac{\varepsilon}{4}-\frac{\|x-y\|}{2} .
\end{aligned}
$$

An immediate consequence of Corollary $1(\mathrm{i}) \Leftrightarrow($ iii), Lemma 1 , formula (1) and Theorem 1 is

COROLLARY 2. The following properties of a given norm of $X$ are equivalent:

(i) $\|\cdot\|$ is rough.

(ii) There is an $\varepsilon>0$ such that for every $x \in S_{X}$ and $\delta>0$ there exist $y, u \in S_{X}$, $\|y-x\|<\delta$, such that either $\tau(y, u) \geqslant \varepsilon$ and $\tau(y,-u) \geqslant \varepsilon$ or $\tau(x, u) \geqslant \varepsilon$ and $\tau(y,-u) \geqslant \varepsilon-\delta$.

We recall $[10$, p. 976$]$ that a normed linear space $E$ has property $(\mathscr{T})$ if each closed bounded convex set $K \subset E$ is the intersection of the closed balls containing it. We need the following result of [10, Lemma 4.1].

LEMMA $2[10$, p. 979]. Suppose that the normed linear space $E$ has property (T). If $f \in S_{E^{*}}$ and $0<\varepsilon<1$, there exist $\delta(\varepsilon)>0$ and $x \in S_{E}$ such that $y \in S_{E} \cap B_{E}(x, \delta)$ implies $A(y) \subset B_{E^{*}}(f, \varepsilon)$.

COROllary 3. If $X$ has property $(\mathcal{T})$, then the norm of $X$ is not rough.

Proof. Use Theorem 1 and Lemma 2.

Though Lemma 2 property characterizes property $(\mathcal{T})[5$, p. 114], the converse to Corollary 3 is not always true. Indeed, it is known [12, p. 178] that if a Banach space $X$ is reflexive, then $X$ is an Asplund space (i.e., every continuous convex function defined on an open convex subset of $X$ is Frechet differentiable on a dense $G_{\delta}$ subset of its domain); by [7, p. 344] $X$ is an Asplund space if and only if $X$ does not admit any equivalent rough norm. In particular, any norm on a 2-dimensional Banach space is not rough. On the other hand, by [10, p. 981] a 2-dimensional Banach space $X$ has property $(\mathcal{T})$ if and only if $X$ is smooth.

Due to the terminology, the following result seems surprising. There is a Banach space $X$ whose norm is both rough and smooth.

EXAMPLE. In [10, p. 981] a norm for $m=\left(l^{1}\right)^{*}$ (the space of bounded sequences $\left.y=\left\{y_{i}\right\}\right)$ was defined by $\|y\|=\sup \left|y_{i}\right|+\left(\sum y_{i}^{2} / 2^{i}\right)^{1 / 2}$ and for $x=\left\{x_{i}\right\} \in l^{1}$, $\|x\|=\sup \left\{\sum x_{i} y_{i} \mid y=\left\{y_{i}\right\} \in m,\|y\|=1\right\}$. Then $X=\left(l^{1},\|\cdot\|\right)$ is a smooth space 
[10, p. 981]. Let $\varepsilon<\frac{1}{8}$ and $x \in S_{X}$. It was shown in [10, p. 982] that for any $y \in S_{X^{*}}$ such that $(x, y)=1$, there exists a sequence $\left\{y^{k}\right\} \subset X^{*}$ such that $\left(x, y^{k}\right) \rightarrow 1$, $\left\|y^{k}\right\| \rightarrow 1$ and $\left\|y^{k}-y\right\| \geqslant \frac{1}{8}$. (Note that in the first case in [10, p. 982] it was not used that $y_{i} \rightarrow 0$ but only that $\left|y_{i}\right|<\frac{1}{4}$ for $i>n$.) Now, for $f_{k}, g_{k} \in S_{X^{*}}$ defined by $f_{k}=y, g_{k}=y^{k} /\left\|y^{k}\right\|$, we have $\lim f_{k}(x)=\lim g_{k}(x)=1$ and $\lim \sup \left\|f_{k}-g_{k}\right\|>\varepsilon$, whence by Proposition 1, the norm on $X$ is rough.

We remark also that there is a Banach space having a nonrough norm with no point of Gateaux differentiability [7, p. 341].

The characterization given in Theorem 1, helps us to introduce the following concept dual to rough norm.

Definition 2. A norm of a Banach space $X$ is said to have property $(d r)$ if there is an $\varepsilon>0$ such that for every $f \in \operatorname{Dom}\left(A^{-1}\right)$ and $\delta>0$, there exists $g \in \operatorname{Dom}\left(A^{-1}\right)$, $\|g-f\|<\delta$ with $\operatorname{diam}\left(A^{-1}(f) \cup A^{-1}(g)\right) \geqslant \varepsilon$. When the Banach space is a dual space $X^{*}$ and in the above definition $f$ and $g$ are chosen to be $w^{*}$-continuous, then the dual norm of $X^{*}$ is said to have property $\left(w^{*} d r\right)$.

To prove the next result we need the following extension of Bishop and Phelps theorem given in [2, Theorem 1].

THEOREM 2 [2, p. 181]. Suppose $x \in S_{X}, f \in S_{X^{*}}$ and $|f(x)-1| \leqslant \varepsilon^{2} / 2\left(0<\varepsilon<\frac{1}{2}\right)$. Then there exist $y \in S_{X}$ and $g \in A(y)$ such that $\|f-g\| \leqslant \varepsilon$ and $\|x-y\|<\varepsilon+\varepsilon^{2}$.

THEOREM 3. Let $X$ be a Banach space. Then:

(i) The norm of $X$ has property $(d r)$ if and only if the dual norm of $X^{*}$ is rough.

(ii) The norm of $X$ is rough if and only if the dual norm of $X^{*}$ has property ( $\left.w^{*} d r\right)$.

Proof. Suppose the norm of $X$ has property (dr) and let $\varepsilon>0$ be given by Definition 2. Let $f \in S_{X^{*}}$ and $\delta>0$. By Bishop and Phelps theorem there exists $f_{1} \in \operatorname{Dom}\left(A^{-1}\right)$ such that $\left\|f_{1}-f\right\|<\delta / 2$. By property (dr) there exists $f_{2} \in$ $\operatorname{Dom}\left(A^{-1}\right),\left\|f_{2}-f_{1}\right\|<\delta / 2$ with $\operatorname{diam}\left(A^{-1}\left(f_{1}\right) \cup A^{-1}\left(f_{2}\right)\right) \geqslant \varepsilon$. Let $x, y \in A^{-1}\left(f_{1}\right)$ $\cup A^{-1}\left(f_{2}\right)$ such that $\|x-y\| \geqslant \varepsilon / 2$. Regarding $X$ as a subspace of $X^{* *}$ we have for $F \in A(f)$ that $\varepsilon / 2 \leqslant\|x-y\| \leqslant\|x-F\|+\|F-y\|$, and so we can suppose that $\|x-F\| \geqslant \varepsilon / 4$. Since $\left\|f_{i}-f\right\|<\delta, i=1,2$ and $x \in A\left(f_{i}\right)$ for some $i=1$ or 2 , it follows that $\operatorname{diam}\left(A(f) \cup A\left(f_{i}\right)\right) \geqslant \varepsilon / 4$, whence by Theorem 1, the dual norm of $X^{*}$ is rough.

Conversely, suppose the dual norm of $X^{*}$ is rough and let $0<\varepsilon<2$ be given by Proposition 1(ii). Let $f \in \operatorname{Dom}\left(A^{-1}\right)$ and $0<\delta<\varepsilon / 4$. By Proposition 1, there exist $x_{n}, y_{n} \in S_{X}, \lim f\left(x_{n}\right)=\lim f\left(y_{n}\right)=1$ such that $\limsup \left\|x_{n}-y_{n}\right\|>\varepsilon$. Let $n_{0}$ be such that $\left|1-f\left(x_{n_{0}}\right)\right|<\delta^{2} / 8,\left|1-f\left(y_{n_{0}}\right)\right|<\delta^{2} / 8$ and $\left\|x_{n_{0}}-y_{n_{0}}\right\| \geqslant \varepsilon$, and let $x \in A^{-1}(f)$. Then $\varepsilon \leqslant\left\|x_{n_{0}}-y_{n_{0}}\right\| \leqslant\left\|x_{n_{0}}-x\right\|+\left\|x-y_{n_{0}}\right\|$, and so we can suppose that $\left\|x_{n_{0}}-x\right\| \geqslant \varepsilon / 2$. By Theorem 2, there exist $z_{0} \in S_{X}$ and $f_{0} \in A\left(z_{0}\right)$ such that $\left\|z_{0}-x_{n_{0}}\right\|<\delta$ and $\left\|f-f_{0}\right\|<\delta$. Then $\varepsilon / 2 \leqslant\left\|x_{n_{0}}-x\right\| \leqslant\left\|x_{n_{0}}-z_{0}\right\|+\| z_{0}$ $-x \|<(\varepsilon / 4)+\operatorname{diam}\left(A^{-1}(f) \cup A^{-1}\left(f_{0}\right)\right)$, and so $\operatorname{diam}\left(A^{-1}(f) \cup A^{-1}\left(f_{0}\right)\right) \geqslant \varepsilon / 4$, i.e., the norm of $X$ has property (dr).

Since (ii) is obvious, this completes the proof.

REMARK 2. By the above proof of (i) it follows that the norm of $X$ has property (dr) if and only if there exists an $\varepsilon>0$ such that for every $f \in \operatorname{Dom}\left(A^{-1}\right)$, 
$x \in A^{-1}(f)$ and $\delta>0$, there exists $g \in \operatorname{Dom}\left(A^{-1}\right),\|f-g\|<\delta$ with

$$
\sup _{y \in A^{-1}(g)}\|x-y\| \geqslant \varepsilon .
$$

There exists a strictly convex Banach space $X$ whose norm has property (dr). Indeed, let $\|\cdot\|$ be the norm on $l^{1}$ defined in the above example. Then $c_{0}$ has an equivalent norm whose dual norm on $c_{0}^{*}=l^{1}$ is $\|\cdot\|$. Let $X$ be $c_{0}$ endowed with this equivalent norm. Then $X^{*}$ being smooth, $X$ is strictly convex and the norm of $X^{*}$ being rough, by Theorem 3(i), the norm of $X$ has property (dr).

DEFINITION 3 [7, p. 337]. A norm of a Banach space $X$ is said to be strongly rough if there is an $\varepsilon>0$ such that for every $x \in S_{X}$ there is an element $u \in S_{X}$ with $\tau(x, u)+\tau(x,-u) \geqslant \varepsilon$.

Remark 3. By [7, Proposition 2(i) $\Leftrightarrow$ (ii)] it follows that $\|\cdot\|$ is strongly rough if and only if there is an $\varepsilon>0$ such that for every $x \in S_{X}$, diam $A(x) \geqslant \varepsilon$.

REMARK 4. With a proof similar with that of Corollary 2 , one can show that the following properties of a given norm of $X$ are equivalent:

(i) $\|\cdot\|$ is strongly rough.

(ii) There is an $\varepsilon>0$ such that for every $x \in S_{X}$ there exists $u \in S_{X}$ with $\tau(x, u) \geqslant \varepsilon$ and $\tau(x,-u) \geqslant \varepsilon$.

(iii) There is an $\varepsilon>0$ such that for every $x \in S_{X}$ there exist $f_{1}, f_{2} \in A(x)$ with $\left\{z \in S_{X} \mid f_{1}(z) \geqslant \varepsilon\right\} \cap\left\{z \in S_{X} \mid f_{2}(z) \leqslant-\varepsilon\right\} \neq \varnothing$.

We want now to introduce a concept dual to strongly rough norm. As we shall see, this is far from being satisfactory.

Definition 4. A norm of a Banach space $X$ is said to have property (dsr) if there is an $\varepsilon>0$ such that for every $f \in \operatorname{Dom}\left(A^{-1}\right), \operatorname{diam}\left(A^{-1}(f)\right) \geqslant \varepsilon$. When the Banach space is a dual space $X^{*}$ and in the above definition $f$ is chosen to be $w^{*}$-continuous, then the dual norm of $X^{*}$ is said to have property ( $w^{*} d s r$ ).

It is obvious that the norm of $X$ is strongly rough if and only if the dual norm of $X^{*}$ has property ( $w^{*}$ dsr). A result similar to Theorem 3(i) is no longer true, since for example the space $c_{0}$ endowed with the usual norm has property (dsr) while the dual norm of $c_{0}^{*}$ is not strongly rough. We do not have an example of a Banach space $X$ with the following two properties: the norm of $X$ has not the property (dsr) and the dual norm of $X^{*}$ is strongly rough.

For each Banach space $X$, let $\varepsilon_{X}=\sup \left\{\varepsilon \mid \operatorname{diam} A(x) \geqslant \varepsilon\right.$ for each $\left.x \in S_{X}\right\}$. Then clearly this supremum is attained, $0 \leqslant \varepsilon_{X} \leqslant 2$, and we have

$$
\varepsilon_{X}=\inf \left\{\operatorname{diam} A(x) \mid x \in S_{X}\right\} .
$$

The known examples of spaces are with $\varepsilon_{X}=0$ or 2 ; these are not the only cases as the next result shows.

THEOREM 4. For each $\lambda, 0<\lambda<2$, there exist Banach spaces $X$ with $\varepsilon_{X}=\lambda$.

Proof. Let $E=l^{1}(S), S$ uncountable, endowed with the usual norm. Let $f_{0}=$ $\left(\alpha_{s}\right)_{s \in S} \in E^{*}$, where $\alpha_{s_{0}}=1$ for some fixed $s_{0} \in S$ and $\alpha_{s}=0$ for $s \neq s_{0}$. Choose $\beta$ such that $1-(\lambda / 2)<\beta<(2 / \lambda)-(\lambda / 2)$ and let $C$ be the convex hull of $B_{E^{*}}, B_{E^{*}}\left(\beta f_{0}, \lambda / 2\right)$ and $B_{E^{*}}\left(-\beta f_{0}, \lambda / 2\right)$. Then $C$ is a symmetric $w^{*}$-compact convex 
body of $E^{*}$, and it induces an equivalent norm on $E$ by $\|x\| \|=\sup \{f(x) \mid f \in C\}$ for each $x \in E$. We have $\|x\| \leqslant\|x\|\|(\beta+\lambda / 2)\| x \|, x \in E$. Let $X=(E,\|\| \cdot\|\|)$. Then for each $x \in S_{X}$, the set $A_{X}(x)$ intersects either the set $B_{E^{*}} \cup B_{E^{*}}\left(\beta f_{0}, \lambda / 2\right)$ or $B_{E^{*}} \cup B_{E^{*}}\left(-\beta f_{0}, \lambda / 2\right)$. Suppose $A_{X}(x)$ intersects $B_{E^{*}} \cup B_{E^{*}}\left(\beta f_{0}, \lambda / 2\right)$ (the other case is similar). If $A_{X}(x) \cap B_{E^{*}} \neq \varnothing$ then $A_{E}(x) \subset A_{X}(x)$, and for $f_{1}, f_{2} \in A_{E}(x)$ we have $2\left\|f_{1}-f_{2}\right\| /(2 \beta+\lambda) \leqslant\left\|f_{1}-f_{2}\right\|$. Since $\operatorname{diam}_{E^{*}} A_{E}(x)=2$, it follows $4 /(2 \beta+\lambda) \leqslant \operatorname{diam}_{X^{*}} A_{X}(x)$, whence by our assumption on $\beta$ we have $\operatorname{diam}_{X^{*}} A_{X}(x) \geqslant \lambda$. If $A_{X}(x) \cap B_{E^{*}}\left(\beta f_{0}, \lambda / 2\right) \neq \varnothing$ and $A_{X}(x) \cap B_{E^{*}}=\varnothing$, then $A_{X}(x) \subset B_{E^{*}}\left(\beta f_{0}, \lambda / 2\right)$. Since for each $f=\left(\eta_{s}\right)_{s \in S} \in E^{*}$ with $\eta_{s_{0}}=0$ we have $\|f\|=\|f\| \|$, it follows $\operatorname{diam}_{X^{*}} A_{X}(x)=\lambda$. Note that this last case happens e.g., for $x=\left(\xi_{s}\right)_{s \in S} \in S_{X}$ where $\xi_{s_{0}}=2 /(2 \beta+\lambda)$ and $\xi_{s}=0$ for $s \neq s_{0}$. Therefore we have $\varepsilon_{X}=\lambda$.

We conclude this note with some questions.

(1) Is it true that for any Banach space $X$ with $0<\varepsilon_{X}<2$, there exists an equivalent norm for which the corresponding $\varepsilon_{X}$ equals 2? When $\varepsilon_{X}=0$ such a question has in general a negative answer, since $[9,7$, p. 343] if there is an equivalent smooth norm, then there is no equivalent strongly rough norm. On the other hand, in [7, p. 346] the space $X=m$ (which has no equivalent smooth norm [3, p. 522]) has been renormed in such a way that $\varepsilon_{X}=2$. So we can ask whether this is not a general phenomenon, i.e., whether for any Banach space $X$ with no equivalent smooth norm, there is an equivalent norm such that the corresponding $\varepsilon_{X}$ equals 2 .

(2) There exist spaces $X$ with the property that diam $A(x)=\varepsilon_{X}$ for all $x \in S_{X}$. For example, $l^{\prime}(S), S$ uncountable, has this property with $\varepsilon_{X}=2$, and the smooth spaces with $\varepsilon_{X}=0$. Is there a Banach space $X$ such that diam $A(x)=\varepsilon_{X}$ for all $x \in S_{X}$ and $0<\varepsilon_{X}<2$ ? If the answer is affirmative, is it true that for any $\lambda$, $0<\lambda<2$, there exists a Banach space $X$ such that $\varepsilon_{X}=\lambda$ and diam $A(x)=\varepsilon_{X}$ for all $x \in S_{X}$ ?

We want to thank Professor V. Zizler for helpful and stimulating conversations related to the subject matter of this paper. We also want to thank the referee for helpful suggestions.

\section{REFERENCES}

1. R. Anantharaman, T. Lewis and J. H. M. Whitfield, Smoothability, strong smoothability and dentability in Banach spaces (to appear).

2. B. Bollobás, An extension to the theorem of Bishop and Phelps, Bull. London Math. Soc. 2 (1970), $181-182$.

3. M. M. Day, Strict convexity and smoothness of normed spaces. Trans. Amer. Math. Soc. 78 (1955), 516-528.

4. N. Dunford and J. T. Schwartz, Linear operators. Part I. General theory, Pure and Applied Mathematics, vol. 7, Interscience, New York and London, 1958.

5. J. R. Giles, D. A. Gregory and B. Sims, Characterisation of normed linear spaces with Mazur's intersection property, Bull. Austral. Math. Soc. 18 (1978), 105-123.

6. J. Hagler and F. Sullivan, Smoothness and weak* sequential compactness, Proc. Amer. Math. Soc. 78 (1980), 497-503.

7. K. John and V. Zizler, On rough norms on Banach spaces, Comment. Math. Univ. Carolinae 19 (1978), 335-349.

8. J. Kurzweil, On approximation in real Banach spaces, Studia Math. 14 (1954), 214-231. 
9. E. B. Leach and J. H. M. Whitfield, Differentiable functions and rough norms on Banach spaces, Proc. Amer. Math. Soc. 33 (1972), 120-126.

10. R. R. Phelps, A representation theorem for bounded convex sets, Proc. Amer. Math. Soc. 11 (1960), 976-983.

11. F. Sullivan. Dentahility, smoothability and stronger properties in Banach spaces, Indiana Univ. Math. J. 26 (1977), 545-553.

12. S. L. Troyanski, On locally uniformly convex and differentiable norms in certain non-separable Banach spaces. Studia Math. 37 (1971), 173-180.

13. J. H. M. Whitfield, Rough and strongly rough norms on Banach spaces, Abstracta of the Seventh Winter School Praha, 1979.

Department of Mathematics, INCREST, B-DUl, PACII 220, 79622 Bucharest, Romania 